\title{
The role of detours in individual human navigation patterns of complex networks
}

\author{
András Gulyás ${ }^{1,3, *}$, József Bíró ${ }^{1}$, Gábor Rétvári ${ }^{1}$, Márton Novák ${ }^{1}$, Attila Körösi ${ }^{1}$, Mariann \\ Slíz ${ }^{2}$, and Zalán Heszberger ${ }^{1,3}$
}

${ }^{1}$ MTA-BME Information Systems Research Group, Budapest University of Technology and Economics, H-1117

Budapest, Magyar tudósok krt. 2, Hungary

${ }^{2}$ Eötvös Loránd University, Institute of Hungarian Linguistics and Finno-Ugric Studies, H-1088 Budapest, Múzeum

krt. 4/A, Hungary

${ }^{3}$ Supported by the János Bolyai Fellowship of the Hungarian Academy of Sciences

*Corresponding author: András Gulyás (gulyas@tmit.bme.hu)

\begin{abstract}
Despite their importance for public transportation, communication within organizations or the general understanding of organized knowledge, our understanding of how human individuals navigate in complex networked systems is still limited owing to the lack of datasets recording sufficient amount of navigation paths of individual humans. Here, we analyze 10587 paths recorded from 259 human subjects when navigating between nodes of a complex word-morph network. We find the clear presence of systematic detours organized around individual hierarchical scaffolds guiding navigation. Our dataset is the first enabling the visualization and analysis of scaffold hierarchies whose presence and role in supporting human navigation is assumed in existing navigational models. By using an information theoretic argumentation, we argue that taking short detours following the hierarchical scaffolds is a clear sign of human subjects simplifying the interpretation of the complex networked system by an order of magnitude. We also discuss the role of these scaffolds in the phases of learning to navigate a network from scratch.
\end{abstract}

\section{Introduction}

Everyday life is full of complex networked systems humans navigate recurringly on a daily basis (e.g. traveling between locations in a city using public transportation). The available navigational datasets ${ }^{1-4}$ and models ${ }^{3-10}$ considering networked systems, mostly target to uncover the average properties of a group of subjects and capture collective human behavior. Thus existing datasets do not have sufficient data or appropriate tracing methods permitting the analysis of long-term individual patterns. Here, we analyze the results of an experiment with human subjects solving navigational tasks in a complex word-morph network. We have recorded on average 40.9 timely-ordered paths from 259 subjects and more than 200 paths from 9 subjects which makes the analysis of individual human navigation patterns possible. The nodes of the word-morph network are English words which are connected if they differ in only a single letter. In this large and complex network, human subjects are given navigational tasks, i.e. to reach a destination word from a starting word by changing only one letter at a time, while still having meaningful words at intermediate states. Figure 1a shows a sample fragment of the word-morph network and two solutions (a shortest path and a human path) of the task with the starting word "yob" and destination word "way".

Network theoreticians across many disciplines ${ }^{11-15}$ argue that the shortest path, i.e. the path containing the minimal number of intermediate steps in a network, between a source and a destination is a usable approximation of the real paths between them. In contrast with these results, our study shows that humans subjects frequently apply detours, even in the long run. Our main finding is that these detours are the consequences of how an individual interprets a complex networked system on its own level. We show that people tend to build up a significantly simpler representation of the word-morph network in the form of a hierarchy in their minds. These hierarchies are then used as scaffolds to the paths in the network. As a result, real paths will be somewhat longer than the shortest alternatives, but the detours will be characteristic to the individual taking them, as no two may abstract away the same hierarchy of the network. Although there are existing models assuming latent hierarchical scaffolds aiding navigation ${ }^{6,10,16-19}$, this is the first study processing sufficient individual human navigation data to visualize and analyse these individually created hierarchies.

Although this study concentrates on a networked system, the underlying problem of human navigation in the word-morph network seems even more interesting in the light that current explanations of physical navigation tend to apply models considering the graph-like abstraction of the surrounding physical environment. In fact, there is an ongoing debate about if we 
build a detailed cognitive map or a much simpler cognitive graph of the possible physical choice points ${ }^{20,21}$ inside our head. Furthermore, recent studies reported major correlations between navigation and learning skills of humans ${ }^{22,23}$ while others go even further and investigate the possibility that navigation in cognitive spaces may lie in the core of any form of organized knowledge and thinking ${ }^{24-26}$. The word-morph network is a special mixed system over which navigation relies strongly on domain-general mechanisms since both spatial, manifested in the Hamming distance between words, and cognitive dimensions, i.e. the function and the meaning of the words, contribute to the formation paths. Thus, the identification of individual scaffolds guiding human navigation in the word-morph network may contribute to the better understanding of how humans structure, encode and navigate through cognitive spaces.

The empirical confirmation of individual scaffold hieararchies may also help to resolve known anomalies in modeling human navigation behaviour in networks. Human paths over networks are reported to exhibit non-negligible memory ${ }^{27-29}$, which leads to problems when applying first-order Markov chains to approximate paths in spreading dynamics and community detection ${ }^{27}$. Individual scaffold hierarchies explain the source of these anomalies as the next step of hierarchically guided paths clearly depends on nodes visited previously by the given individual. Building on the assumption of hierarchical scaffolds behind network paths we may able to refine higher-order Markov models and bring us closer to the better understanding of how real systems are organized and function.
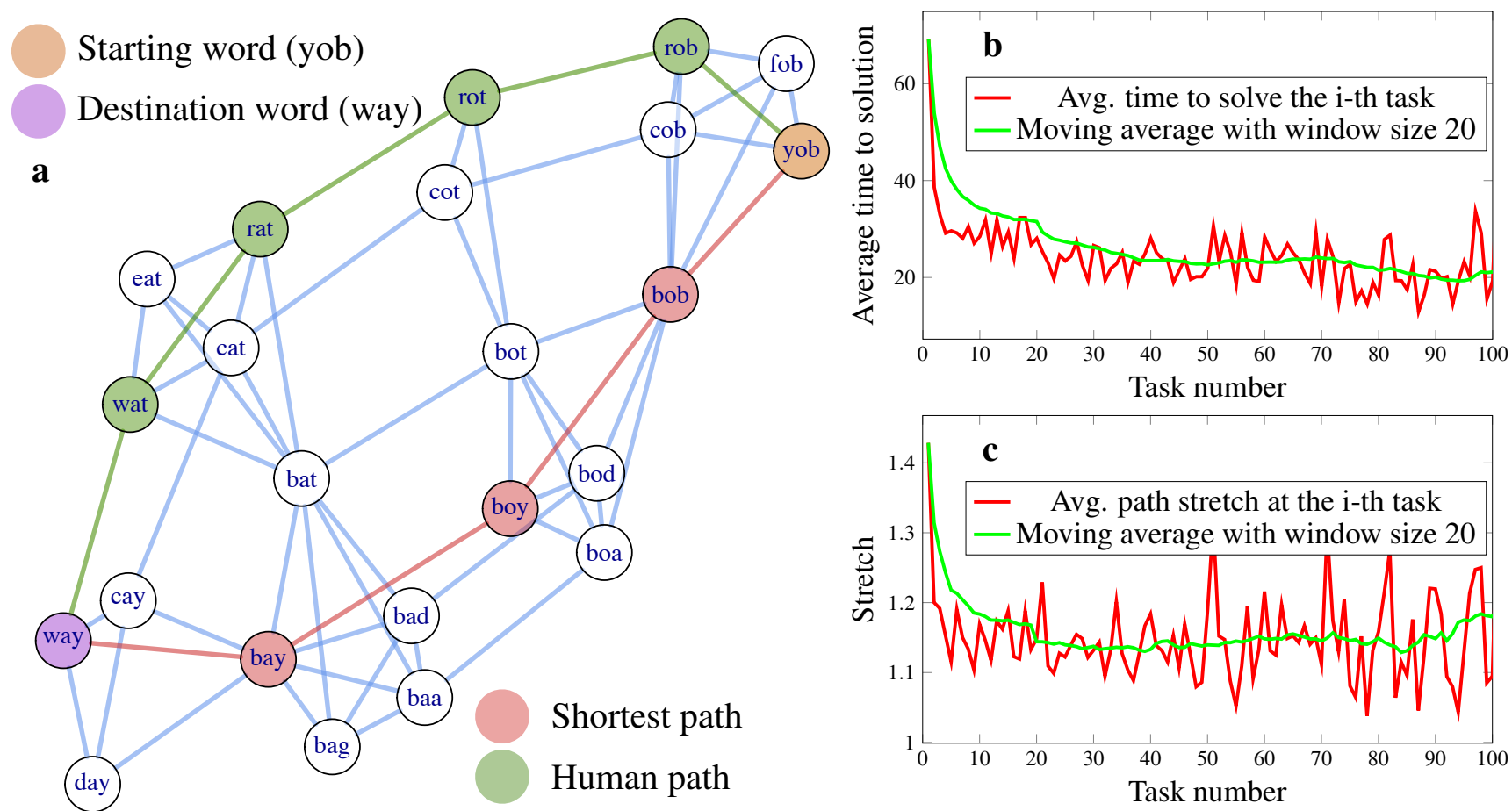

Figure 1. An example and high-level statistics of our navigation experiment. Panel (a) shows a sample section of the network of three-letter English words, in which two words are connected if they differ only in a single letter. When human subjects solve a navigation task, they come up with a path from a randomly given starting word to a destination word by changing only a single letter at a step such that they always get a valid intermediate English word. The red and green paths show a shortest and slightly detoured human solution from "yob" to "way". Panel (b) presents the average time it takes for human subjects to solve the n-th task in a row, while panel (c) shows the stretch of the human paths, i.e. the ratio of the length of the paths found by human subjects and the length of the shortest possible path in the word-morph network. While the average time to solve a task clearly decreases with the number of tasks solved, the stretch of the solutions stabilizes between 1.2 and 1.1. This suggests that human subjects develop a specific strategy in the first few rounds, but after a few tens of solved tasks their strategy is not improved any further in terms of length. So they have a simplified interpretation of the network and they find their paths through that, only slightly faster as time goes by.

We discuss that navigational scaffold hierarchies may boost up the learning process to navigate in the word-morph network and reduce the memory requirement of navigation by an order of magnitude. Moreover, identifying the individual scaffold hierarchies as the enablers of memory-efficient navigation in the word-morph network is of particular importance since this may promote to uncover navigational schemes in other complex networked systems considering not only humans. Similar detours have been identified in measurements capturing collective behaviour in networks from diverse areas of life. Gao et al. 
showed that the paths of packets going through the internet are also detoured in a non-negligible extent ${ }^{30}$ and they show that the hierarchical policies of internet packet routing may be responsible for the major proportion of the inflation. Detours have been identified in road networks by Zhu et al. ${ }^{31}$ and in cattle pen systems by Grandin ${ }^{32}$, while similar phenomena was reported in airport $^{10,27}$ and brain networks ${ }^{10,33}$ too.

\section{Results}

For our experiment we have designed a word-morph game application for smartphones ${ }^{34}$ (see Methods for details). The application collected 19828 paths from 259 human subjects navigating in the word-morph network. After cleaning the data by removing tasks that were either unfinished, contained loops or taking extraordinary long time ( $>300$ seconds) to complete, our working dataset of paths was reduced to 10857 paths. The word-morph network is a complex network which is impossible for a human subject to keep fully in mind with its 1008 nodes and 8320 edges. The average degree, the diameter and the clustering coefficient of the network is $16.39,9$ and 0.44 respectively. To get a high-level impression about the performance of human navigation we have plotted the average time needed to solve the n-th task in a row in Figure 1-b. We can see that after a few of initial rounds human subjects find a solution in around 30 seconds on average and from there on they slowly improve to around 20 seconds after solving 100 tasks. It is a kind of astonishing in itself, that after a few rounds people can find paths in this complex maze very efficiently. Strikingly, the improvement in time doesn't imply that the paths found are also shorter. In Figure 1-c, the stretch of human solutions is shown compared to the shortest paths. The stretch of a path $P$ is computed as the ratio of the length of $P$ and the length of the shortest path between identical starting and destination words. In the example of Figure 1-a, the stretch of the human path (green) is $\frac{5}{4}=1.25$ compared to the shortest possible path (red). Figure 1-c shows that although human subjects improve in terms of the time needed to solve a task, the stretch of the paths they find stabilizes slightly below 1.2. Thus the length of the human paths seem not to converge to the length of the shortest path (i.e. to stretch 1) and they always include some detours. A plausible explanation for this is that human subjects develop some kind of sub-optimal strategy through the course of the game and use this strategy to solve upcoming tasks. The improvement in time only means that the application of the same strategy becomes more and more effective. But how can we characterize the strategy in use?

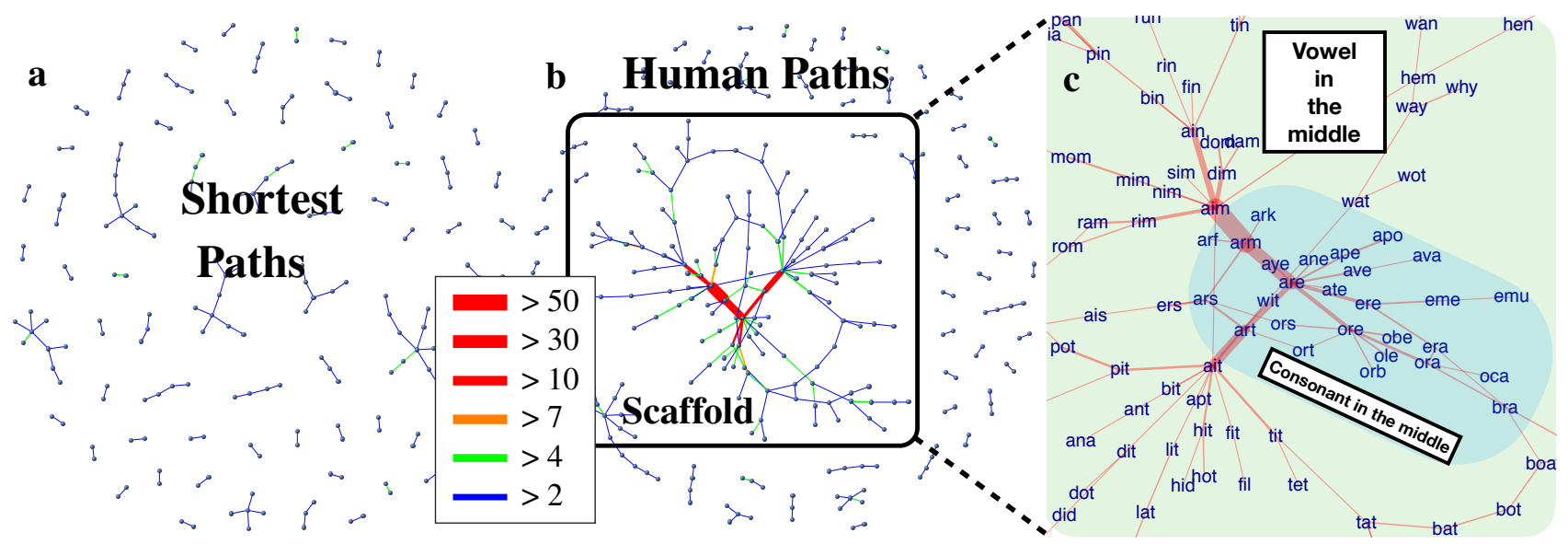

Figure 2. Structures behind human paths and shortest paths. Panel (a) shows how many times an edge is crossed after solving 1000 random tasks by using the shortest path between the source and target word. The almost homogeneous distribution of edge crossings suggests that the entity using these paths, does not have any form of understanding or interpretation of the word-morph network, conversely, it mechanically picks paths. Human paths are quite the contrary. Panel (b) shows the edge crossings of a human subject when solving the same 1000 random tasks. Human solutions appear to be highly structured suggesting that humans possess a characteristic concept of the word-morph network. The structure is very close to a pure hierarchy. There is a clear scaffold that guides navigation, consisting of red, orange and green edges with a high number of crossings. This scaffold shows that human subjects tend to simplify the problem and form a simpler, systematic although not necessarily optimal strategy. From the sides of the network, from where a navigation task starts, humans tend towards their scaffold where they switch to other sides of the network. It is quite specific how this particular scaffold is built up. Panel (c) shows the words in the middle of the scaffold. "Aim", "art", "arm" and "are" words where consonants and vowels can be changed very effectively. In this case, the scaffold is used to switch between regimes of the network based on the location of vowels and consonants.

Panels $\mathbf{a}$ and $\mathbf{b}$ in Figure 2 shows how differently an algorithm implementing shortest paths and a human subject use the 

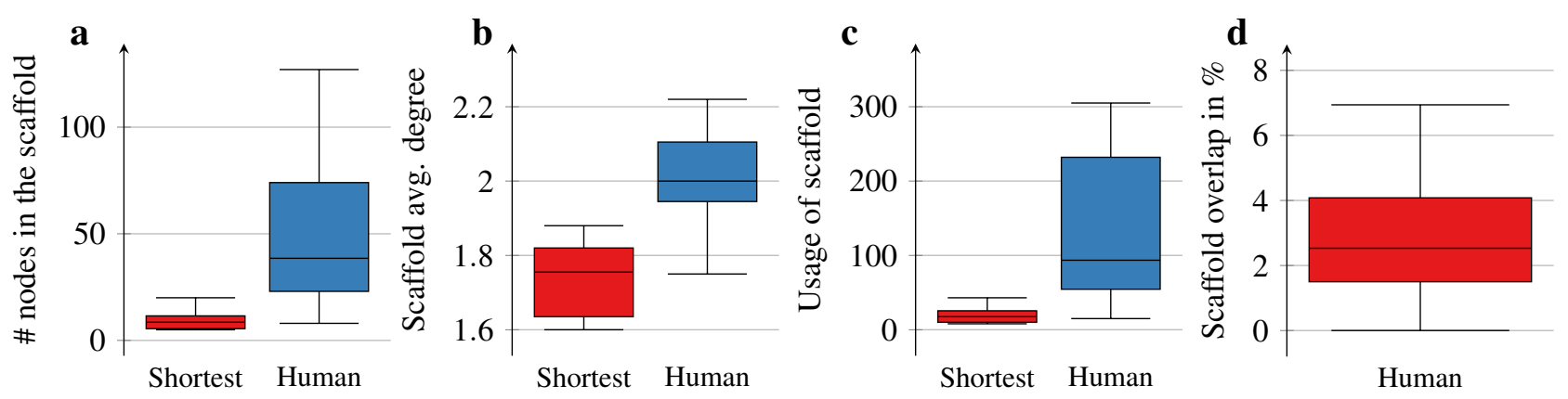

Figure 3. Properties of individual human scaffolds. Panel (a) shows the size of the human scaffolds compared to the shortest path case. Human subjects' behavior clearly deviates from the shortest path algorithm as they form sizeable navigational scaffolds compared to shortest paths. The average degree of the scaffolds is closely around 2 as shown in panel (b), thus the structure is very close to trees. Panel (c) confirms that the scaffold is heavily used by human subjects when completing the navigation tasks. Panel (d) shows, that the individual human scaffolds are indeed "individual" as the observed overlaps between the subjects' scaffolds is only $2.6 \%$ on average.

word-morph network to solve the navigational tasks. The plots show only edges traversed more than two times in the course of solving 1000 tasks. In the case of the shortest path algorithm, the usage of edges is homogeneous. The algorithm has no clear concept or deeper interpretation of the word-morph network, thus picks the paths mechanically without any sign of favoring specific regions of the network. Human subjects behave quite differently. Humans seem to have a clear concept of the network. They structure the network in a subjective manner by identifying various regions and put a larger emphasis on nodes and edges connecting these regions. The clear sign of this is that from human solutions a hierarchical scaffold structure is formed (see Figure 2-b for an example). For capturing this behavior we have investigated subjects having more than 200 completed navigation tasks ( 9 subjects qualified for this). For these subjects, we have processed all the solutions of the navigational tasks and assigned weights to the edges of the word-morph network reflecting how many times they have been used in the solutions. We have dropped the edges having used less than 2 times. From the remaining graph we took the largest component as the scaffold. In $90 \%$ of the cases, the scaffolds of the human subjects were at least two times bigger in size compared to the random case, but in the majority of the cases the human scaffolds were found to be an order of magnitude bigger (see Panel a in Figure 3).

Panel $\mathbf{b}$ of Figure 3 shows that the average degree of the scaffolds is around 2 in case of all the subjects. This means that the scaffolds are tree-like connected sub-networks of the original word-morph network. This result is fully in-line with the assumptions of existing hierarchical human navigational models $6,16,17,19$. Compared to shortest paths the edges of the scaffolds are heavily used by the subjects (see Figure 3-c) with a very specific usage pattern. The scaffold has a definite core of a few nodes between which the usage of the edges can go above 50 in the particular example of Figure 2-b. This core behaves as a switching device among different parts of the network and abstracts the concept of the individual about the structure of the whole network. The scaffold is built up in a hierarchical, tree-like fashion as edge utilization clearly drops when receding from the core. In the course of navigating between words, subjects use the scaffold as a guiding framework. Figure 2-c shows the words residing in the scaffold. In this example, the network is clearly divided into regions based on the position of consonants and vowels in the words and the core words are picked by the human subject in order to switch effectively among these regions. Our results show that although these individual scaffolds may have some similarities, every subject used a fairly unique set of nodes and edges forming their own hierarchical scaffolds (see supplementary Figure 1 for additional examples of personal scaffolds). This finding is readily supported by Figure 3-d, which shows the percentage of overlap between all possible pairs of scaffolds. The overlap for scaffolds $i$ and $j$ is computed as $\frac{E\left(G_{i}\right) \cap E\left(G_{j}\right)}{E\left(G_{i}\right) \cup E\left(G_{j}\right)}$, i.e. the ratio of edges present in both scaffolds and the edges in the union of the scaffolds, thus a network's overlap with itself is practically $100 \%$. One can see, that in the case of the scaffolds of our subjects the average of the overlaps is very small, around $2.6 \%$ and in $90 \%$ of the cases do not exceed $7 \%$.

\section{Discussion}

The identification of the individual scaffold hierarchies as core switching devices in the human interpretation of the word-morph network poses an intriguing question: Why do we use them even after mastering ourselves in the navigation task? Why do we settle with sub-optimal paths through these scaffold hierarchies and not combat for shorter paths? Recall, that detours in our subjects' paths persisted even after completing 100 navigation tasks. We argue that the reason behind this is related to our capabilities of information encoding and processing. In short, we build scaffold hierarchies while being satisfied with 
sub-optimal paths because this way we don't have to process every bit of information about a large and complex system and we can get away with an order of magnitude simpler interpretation. For showing this we will use the following minimalist information-theoretic model inspired by our results above. The word-morph network is represented by a graph $G(N, E)$ defining its nodes $N$ and edges $E$. For modeling human behavior we use a simple tree hierarchy as a scaffold for navigation. The construction of the hierarchy goes by picking the node with the highest closeness centrality ${ }^{35}$ and building the breadth-first search (BFS) tree emanating from it. This BFS tree will be used as the scaffold. Inspired by the information exchange algorithm well-fitted for hierarchically structured organizations ${ }^{16}$, we define human navigation based on the scaffold hierarchy as follows: (i) if the destination node is below the current node or its neighbors in the hierarchy, then we step to its closest superior or the destination itself provided that the destination and the current nodes are connected, (ii) if the destination node is not below the current node in the hierarchy, then we step to the current node's direct superior in the hierarchy. With an analogy, this simple navigation mechanism captures that if somebody is my subordinate in the hierarchy or someone's subordinate that I know, then I know who is the closest to it among my acquaintances. If I know nothing about the target, then I turn to my direct superior.

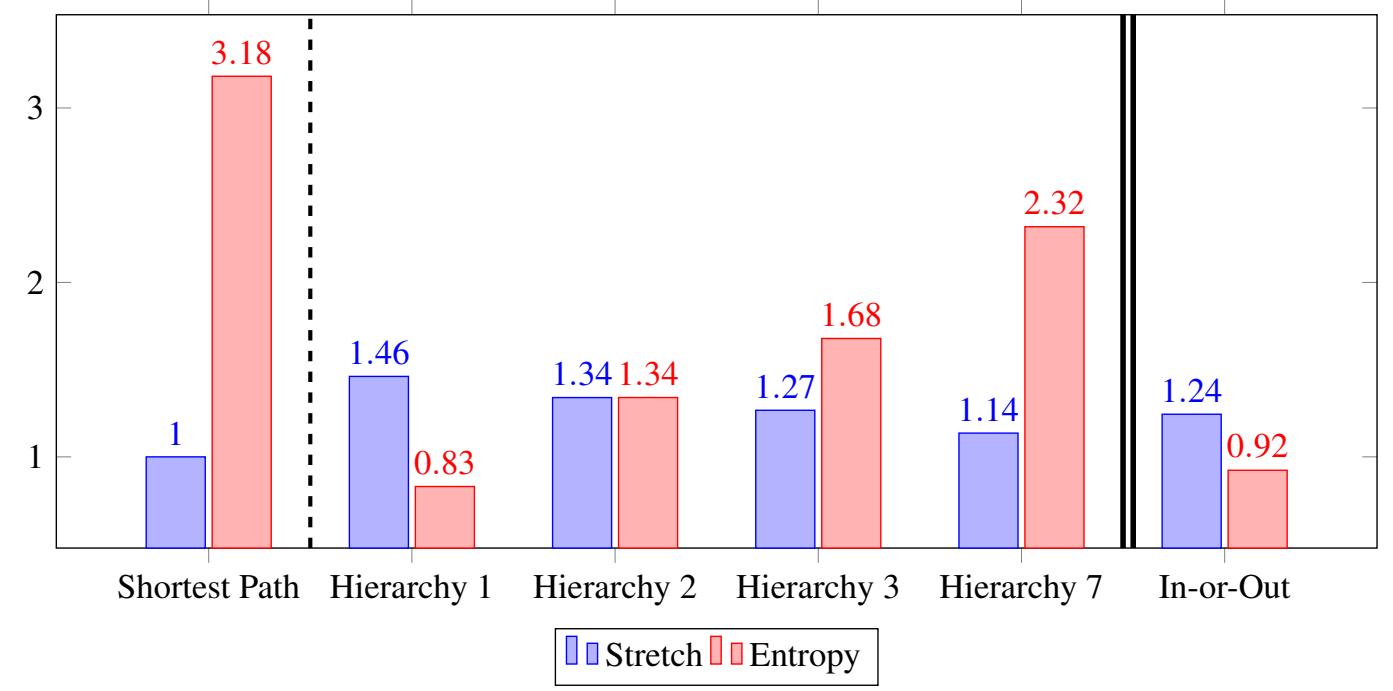

Figure 4. Comparison of stretch and entropy of various paths. Shortest paths clearly have a stretch of 1 , but this optimality comes at a price of high entropy, i.e. high memory requirement to store. Hierarchy 1-7 shows the very efficient stretch-entropy tradeoff if we memorize only 1-7 uplinks in the most simple BFS hierarchy. The decentralized In-and-Out hierarchy with one direct superior, based on highest closeness centrality, is a sweet spot in this tradeoff space. This simulates the case, when people know all subordinates in the network, but remember only one superior closest to the center of the network. It gives a realistic stretch, but the required entropy is an order of magnitude lower than in the shortest path case.

For characterizing the complexity of implementing the paths provided by the shortest path algorithm and human navigation, we will approximate the required minimum information in every node to decide where to take the next step towards all destinations in the word-morph network. Let's assign positive integers, i.e. 1,2,3 ... as IDs to the nodes of the network. At each node $x$, we can represent the amount of information needed to make the right choice by a node table $T_{x}$. At node $x$, this node table has $|N|-1$ entries (where $|N|$ is the number of nodes in the word-morph network) belonging to all the nodes other than $x$, and each entry contains the ID of a neighbor to take next heading towards a given destination. For example a node table $T_{5}=(1,2,1,2)$ tells us that at node 5 , if we want to go to node $1,2,3,4$ we should take nodes $1,2,1,2$ as next steps respectively. This node table implicitly tells us, that node 5 is connected to nodes 1 and 2 and that, in this example, the network has five nodes. Supplementary Note 1 provides a more detailed example of how to compute these node tables for a concrete network and set of paths. Now tables $T_{x}, \forall x \in N$ contain all the information required to implement the given paths between arbitrary pairs of nodes in the word-morph network. To approximate how many bits of information are needed to store these tables in memory, we compute their empirical Shannon-entropy ${ }^{36}$, defined as $H_{0}\left(T_{x}\right)=\sum_{c \in \Sigma} \frac{n_{c}}{n} \log \frac{n}{n_{c}}$, where $\Sigma$ denotes the set of different numbers in $T_{x}$, while $n$ and $n_{c}$ represents the length of $T_{X}$ and the number of occurrences of each number $c \in \Sigma$ in $T_{x}$ respectively. Then $\frac{\sum_{x \in N} H_{0}\left(T_{x}\right)}{n}$ gives the global per-node entropy to implement the paths. In Supplementary Note 2 we supply asymptotically optimal results for the empirical entropy of some well-known graph families.

In Figure 4 the required information for implementing shortest paths and hierarchical paths in the word-morph network is shown. Shortest paths clearly have a stretch of one, but the price of this is high entropy as around 3.18 bits per node is required to store the shortest paths in the node tables (see the Shortest Path column on the left of Figure 4). Navigation with 


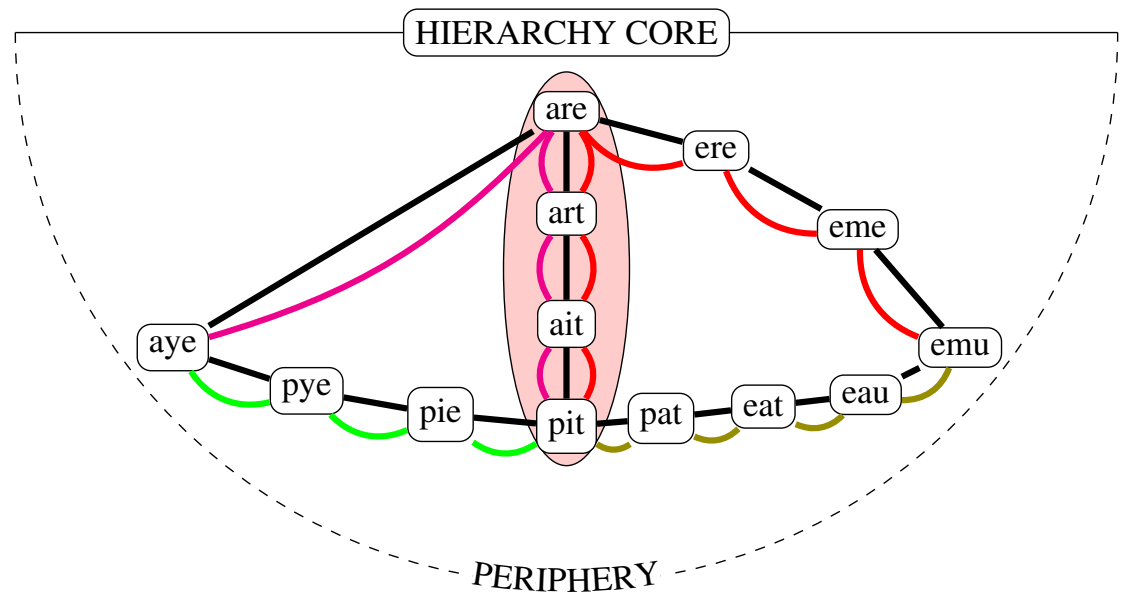

Figure 5. Shortest and hierarchically guided paths in the word network. Learning only the shortest paths between the words pit and aye and between pit and ети makes us conclude that the word aye is 7 nodes away from ети. However, with a hierarchical scaffold a four-node path between aye and ети can be found even though both the paths between pit and aye and between pit and ети are longer through the scaffold than the shortest possible path.

the simple BFS scaffold has an order of magnitude less (around 0.83 bits per node) entropy (see the Hierarchy 1 column of Figure 4), but hierarchically guided paths are much longer, they have a stretch of 1.46 . Recall that our results with human subjects indicate a stretch slightly below 1.2. Hierarchy 2,3 and 7 columns in Figure 4 stand for a slightly modified version of the BFS hierarchy in which we do not have strictly 1 direct superior but can have links to at most 2,3 and 7 superiors in the BFS tree respectively. These hierarchies are not trees anymore, but they are still sparse as the human scaffolds. These modifications readily illustrate that there is a clear tradeoff between stretch and entropy. Having to remember more superiors reduces stretch but surely increases complexity. Nevertheless, with Hierarchy 7, a stretch of 1.14 is achievable for the price of only 2.32 bits of memory per node. These results readily illustrate that even the most rudimentary scaffold guiding navigation can achieve an effective stretch-entropy tradeoff. However, BFS scaffolds are constructed in a centralized fashion and rely upon global information about the network which is not realistic. A more realistic decentralized scaffold with only one direct superior gives a sweet spot in this tradeoff space while computable with local algorithms ${ }^{37}$. In this hierarchy, called In-or-Out, every node's superior is the neighbor lying in the most central location in the network in terms of closeness centrality. This simple, local strategy can give a very low stretch for an order of magnitude less entropy compared to shortest paths. This is because In-or-Out hierarchy is aware of the neighbors' centrality, thus every node's direct superior is a neighbor that is closest on average to any other node in the network. Interestingly, In-or-Out hierarchy's stretch is close to what we have observed with human subjects.

Besides simplifying the process of navigation, scaffold hierarchies can boost learning the structure of a totally unknown network through observing its paths. For showing this we will use a very simple incremental model where every step we show a single path connecting randomly chosen nodes and compare the reconstructed network structure and the efficiency of navigation based solely on the given paths to the original network. Fig. 5 illustrates the steps of this learning process for the cases if we show paths according to shortest or hierarchical scaffolds from the word-morph network. In the first case, we show the shortest paths between the words "aye" and "pit" (green) and between "pit" and "emu" (olive), and based solely on that knowledge one may implicitly deduce a path from "aye" to "emu" taking 6 nodes to traverse. Alternatively, showing paths using a hierarchical scaffold yields somewhat longer paths (red). However, one can see that the newly gained path between "aye" and "emu" adds up to a substantially shorter path requiring only 3 intermediate nodes. On Fig. 6, the integrity and the stretch and entropy footprint of the various learning scaffolds are shown when we continue simulating the learning process to 2000 paths with a computer program (see Methods for details). On panel (a), the size of the giant component in the network reconstructed from the paths is shown as the function or learned paths. The shortest path scaffold provides only very sporadic knowledge about the network in the initial $(0-120)$ learning steps as the size of the giant component hardly grows with the number of learned paths. The most integrated knowledge is provided by the most simple scaffold of Hierarchy 1. On panel (b) we can clearly distinguish between two phases of the learning process. Until around 700 paths the rough exploration of the nodes and possible connections in the network happens. According to the inset of the panel, by the end of this exploration phase, one can connect more than $90 \%$ of all possible node pairs in case of all scaffolds. Using the shortest paths as learning scaffolds, we can find only very long paths in the exploration phase, as the average stretch can go up to even above 3. Interestingly, if paths are picked according to a hierarchical scaffold, we can obtain paths with a lower stretch as the scaffold becomes more and more simple, i.e. 
the number of direct superiors is decreasing. In case of the simplest one-superior case, the stretch is very stable around 1.5. So in the exploration phase, one can learn reasonable paths much faster if paths are given according to a hierarchical scaffold. After the exploration phase, we do not explore new territories of the word-morph network, what we do is only improving our knowledge. In this improvement phase, the shortest path scaffold takes the lead over the hierarchical scaffolds yielding the best stretch values. The price of being better in stretch is paid on entropy as it can be seen in panel (c): The entropy of the scaffolds is similar in the exploration phase, however, as the number of paths learned increases, the entropy of the simplest Hierarchy 1 scaffold starts to decrease substantially, while the shortest path one continues to increase almost linearly.
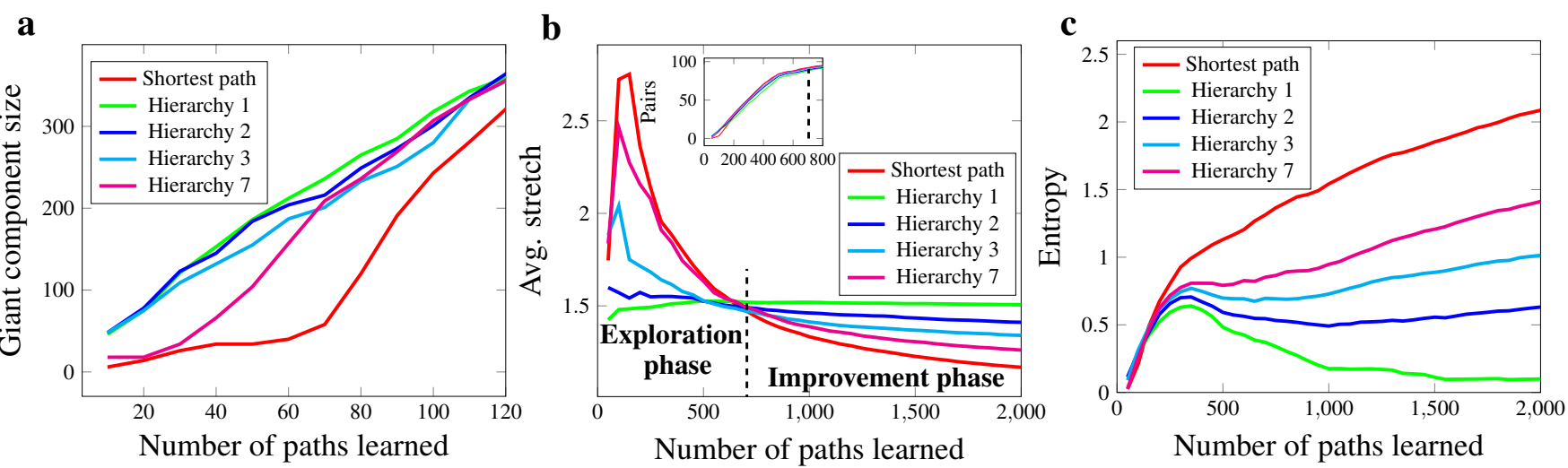

Figure 6. Learning curves in the word-morph network. Panel (a) shows the size of the giant component vs. the number of paths learned according to various learning scaffolds. Using the shortest paths as scaffold gives sporadic knowledge about the network, especially in the initial steps of learning, since the size of the giant component is very low, compared to the other scaffolds. The most integrated knowledge about the network is given by the most simple Hierarchy 1 in the initial steps of learning. The inset of panel (b) shows that after learning only around 700 paths one can infer valid paths between $90 \%$ of all possible node pairs using either the shortest path or hierarchical scaffolds. In this exploration phase, learning based on shortest paths seems to be quite inefficient too, as stretch can go up to even 3. In that phase, the simplest hierarchical scaffold gives the shortest established path on average. Only in the improvement phase, in which no significant new parts of the word-morph network is explored, the relation is reversed. The entropy of the paths is shown in panel (c). The exploration phase shows no difference among the scaffolding schemes, however, in the improvement phase, the entropy of the hierarchical scaffolds is much lower compared to the shortest paths.

\section{Methods}

Data collection - The collection of the results of the navigational tasks in the word-morph network was carried over by a smartphone application called "fit-fat-cat" running on Android platform. The application is available from the Google Play store $^{34}$ and the installation is as easy as any other apps. By using the application, the subjects give their consent to openly use their anonymized data without restrictions. When a subject starts a navigational task, the source and destination words are generated randomly from the all possible three-letter English words. The source and destination words are displayed in a box. Below this box, a list of words the subject visited so far in that particular task is shown. When starting a new task, the list contains only the source word. The subject can enter the consecutive words in a user-friendly manner by using a virtual keyboard of the phone. First, the subject selects the letter to change than choose the new letter with the keyboard. After changing a letter, the app automatically adds the new word to the list. This way the subjects can see which words they have already tackled when solving a particular navigation task. A task may end in three ways. If the subject reached the target word through such one-letter transformations the task is solved. In this case, the word becomes green-colored to show the end of the task. Secondly, the subject can give up the task by pressing the "new game" button. In this case, the subject gets the next task automatically. Finally, the subject can press the "magic wand" button. In this case, a possible (shortest path) solution of the task is shown before starting a new task. No matter how the task is ended, the list of words is anonymously submitted to our database stored in the cloud.

Computer simulations - For investigating the incremental learning of a network via its paths we have written a simulator in the Python programming language. In the beginning, the simulator reads the network $N$. After that, iteratively picks random pairs from the network and compute the shortest and hierarchical paths between them according to the given BFS hierarchy. At each iterative step, the current knowledge about the network is the union nodes and edges contained in the previous iterations. 
So in at step $t$, the knowledge about the network is a graph $G_{t}(V, E)$, then after adding a path $P_{t}$ it is extended to $G_{t+1}=G_{t} \cup P_{t}$. The simulator computes the required entropy and stretch of the paths in $G_{t}$ compared to the shortest paths in $N$ at every 50 step. Data Availability - The data supporting the findings of this study is available from the "fit-fat-cat" public Open Science Framework data repository ${ }^{38}$.

\section{Acknowledgements}

We would like to thank Nora S. Newcombe for the discussions related to human navigation. Project no. 123957, 129589 and 124171 has been implemented with the support provided from the National Research, Development and Innovation Fund of Hungary, financed under the FK_17, KH_18 and K_17 funding schemes respectively.

\section{References}

1. Milgram, S. The small world problem. Psychol. today 2, 60-67 (1967).

2. Dodds, P. S., Muhamad, R. \& Watts, D. J. An experimental study of search in global social networks. Science 301, 827-829, DOI: 10.1126/science.1081058 (2003). http://science.sciencemag.org/content/301/5634/827.full.pdf.

3. West, R. \& Leskovec, J. Human wayfinding in information networks. In Proceedings of the 21 st international conference on World Wide Web, 619-628 (ACM, 2012).

4. Iyengar, S., Zweig, N., Natarajan, A. \& Madhavan, V. A network analysis approach to understand human-wayfinding problem. In Proceedings of the Annual Meeting of the Cognitive Science Society, vol. 33 (2011).

5. Kleinberg, J. M. Navigation in a small world. Nature 406, 845 (2000).

6. Watts, D. J., Dodds, P. S. \& Newman, M. E. Identity and search in social networks. science 296, 1302-1305 (2002).

7. Şimşek, Ö. \& Jensen, D. Navigating networks by using homophily and degree. Proc. Natl. Acad. Sci. 105, 12758-12762 (2008).

8. Adamic, L. A., Lukose, R. M., Puniyani, A. R. \& Huberman, B. A. Search in power-law networks. Phys. review E 64, 046135 (2001).

9. Boguna, M., Krioukov, D. \& Claffy, K. C. Navigability of complex networks. Nat. Phys. 5, 74 (2009).

10. Csoma, A. et al. Routes obey hierarchy in complex networks. Sci. Reports 7, 7243, DOI: 10.1038/s41598-017-07412-4 (2017).

11. Wardrop, J. G. Some theoretical aspects of road traffic research. In Inst Civil Engineers Proc London/UK/ (1952).

12. Watts, D. J. \& Strogatz, S. H. Collective dynamics of 'small-world'networks. Nature 393, 440 (1998).

13. Barabasi, A.-L. \& Oltvai, Z. N. Network biology: understanding the cell's functional organization. Nat. reviews genetics 5, 101 (2004).

14. Montoya, J. M., Pimm, S. L. \& Solé, R. V. Ecological networks and their fragility. Nature 442, 259 (2006).

15. Bullmore, E. \& Sporns, O. Complex brain networks: graph theoretical analysis of structural and functional systems. Nat. Rev. Neurosci. 10, 186 (2009).

16. Dodds, P. S., Watts, D. J. \& Sabel, C. F. Information exchange and the robustness of organizational networks. Proc. Natl. Acad. Sci. 100, 12516-12521, DOI: 10.1073/pnas.1534702100 (2003). https://www.pnas.org/content/100/21/12516.full. pdf.

17. Kleinberg, J. M. Small-world phenomena and the dynamics of information. In Advances in neural information processing systems, 431-438 (2002).

18. Gao, L. \& Rexford, J. Stable internet routing without global coordination. IEEE/ACM Transactions on Netw. (TON) 9, 681-692 (2001).

19. Helic, D., Strohmaier, M., Granitzer, M. \& Scherer, R. Models of human navigation in information networks based on decentralized search. In Proceedings of the 24th ACM conference on hypertext and social media, 89-98 (ACM, 2013).

20. Newcombe, N. S. Individual variation in human navigation. Curr. Biol. 28, R1004 - R1008, DOI: https://doi.org/10.1016/ j.cub.2018.04.053 (2018).

21. Chrastil, E. R. \& Warren, W. H. From cognitive maps to cognitive graphs. PloS one 9, e112544 (2014).

22. Newcombe, N. Harnessing spatial thinking to support stem learning. OECD Educ. Work. Pap. DOI: https://doi.org/https: //doi.org/10.1787/7d5dcae6-en (2017). 
23. Möhring, W., Frick, A. \& Newcombe, N. S. Spatial scaling, proportional thinking, and numerical understanding in 5- to 7-year-old children. Cogn. Dev. 45, 57 - 67, DOI: https://doi.org/10.1016/j.cogdev.2017.12.001 (2018).

24. Bellmund, J. L. S., Gärdenfors, P., Moser, E. I. \& Doeller, C. F. Navigating cognition: Spatial codes for human thinking. Science 362, DOI: 10.1126/science.aat6766 (2018). http://science.sciencemag.org/content/362/6415/eaat6766.full.pdf.

25. Epstein, R. A., Patai, E. Z., Julian, J. B. \& Spiers, H. J. The cognitive map in humans: spatial navigation and beyond. Nat. neuroscience 20, 1504 (2017).

26. Behrens, T. E. et al. What is a cognitive map? organizing knowledge for flexible behavior. Neuron 100, 490-509 (2018).

27. Rosvall, M., Esquivel, A. V., Lancichinetti, A., West, J. D. \& Lambiotte, R. Memory in network flows and its effects on spreading dynamics and community detection. Nat. communications 5, 4630 (2014).

28. Salnikov, V., Schaub, M. T. \& Lambiotte, R. Using higher-order markov models to reveal flow-based communities in networks. Sci. reports 6, 23194 (2016).

29. Singer, P., Helic, D., Taraghi, B. \& Strohmaier, M. Detecting memory and structure in human navigation patterns using markov chain models of varying order. PloS one 9, e102070 (2014).

30. Gao, L. \& Wang, F. The extent of as path inflation by routing policies. In Global Telecommunications Conference, 2002. GLOBECOM'02. IEEE, vol. 3, 2180-2184 (IEEE, 2002).

31. Zhu, S. \& Levinson, D. Do people use the shortest path? an empirical test of wardrop's first principle. PloS one 10 (2015).

32. Grandin, T. Observations of cattle behavior applied to the design of cattle-handling facilities. Appl. Animal Ethol. 6, 19-31 (1980).

33. Avena-Koenigsberger, A. et al. A spectrum of routing strategies for brain networks. PLoS computational biology 15, e1006833 (2019).

34. fit-fat-cat. Smartphone application. https://play.google.com/store/apps/details?id=hu.bme.tmit.lendulet. wordnavigationgame (2016). [Online; accessed 03-20-2019].

35. Bavelas, A. Communication patterns in task-oriented groups. The J. Acoust. Soc. Am. 22, 725-730 (1950).

36. Shannon, C. E. A mathematical theory of communication. Bell system technical journal 27, 379-423 (1948).

37. You, K., Tempo, R. \& Qiu, L. Distributed algorithms for computation of centrality measures in complex networks. IEEE Transactions on Autom. Control. 62, 2080-2094 (2017).

38. Kőrösi, A. et al. fit-fat-cat dataset. Open Sci. Framew. (2018). http://dx.doi.org/10.17605/OSF.IO/JTYVD. 